\title{
Cyclic Fatigue of Different Nickel-Titanium Rotary Instruments: A Comparative Study
}

\author{
L. Testarelli, N.M. Grande, G. Plotino, M. Lendini, G. Pongione, G. De Paolis, F. Rizzo, V. Milana \\ and G. Gambarini*
}

Department of Dental Science, Sapienza University of Rome, Italy

\begin{abstract}
Since the introduction of nickel-titanium alloy to endodontics, there have been many changes in instrument design, but no significant improvements in the raw material properties, or enhancements in the manufacturing process. Recently, a new method to produce nickel-titanium rotary (NTR) instruments has been developed, in an attempt to obtain instruments that are more flexible and resistant to fatigue. NTR instruments produced using the process of twisting (TF, SybronEndo, Orange, CA) were compared to NTR instruments from different manufacturers produced by a traditional grinding process. The aim of the study was to investigate whether cyclic fatigue resistance is increased for TF NTR files. Tests were performed with a cyclic fatigue device that evaluated cycles to failure of rotary instruments inside curved artificial canals. Results indicated that size 06-25 TF instruments showed a significant increase $(\mathrm{P}<.05)$. In the mean number of cycles to failure when compared to the other tested 06-25 NTR. Hence, it can be concluded that size 06-25 TF NTR instruments were found to be significantly more resistant to fatigue than those produced with the traditional grinding process.
\end{abstract}

Key Words: Endodontic instruments, nickel titanium, cyclic fatigue.

\section{INTRODUCTION}

All nickel-titanium rotary (NTR) instrument systems currently on the market are constructed from "Nitonol" an alloy that was developed in 1988 [1]. Endodontic treatment has benefited from the resulting instruments. This is due to a combination of unique mechanical properties of the alloy, innovative file design, greater tapers and a crown down instrumentation procedure. As a result, there are now many systems available commercially, that utilize NTR instruments of different designs and dimensions, to facilitate cleaning and shaping procedures $[2,3]$.

The only concern expressed by dentists using NTR instruments is the fear of fracture during intracanal use, due to excessive torsional and flexural stress $[4,5]$. A study which assessed 7159 discarded NTR instruments, showed that $5 \%$ had fractured during intracanal use, and that $70 \%$ of these fractures were attributed to flexural (cyclic) fatigue [6]. Cyclic fatigue failure is reported to occur unexpectedly without any sign of previous permanent deformation. This occurs due to the alternating tension/compression cycles which instruments are subjected to, when flexed in the region of maximum curvature of the canal [7].

Since the introduction of nickel-titanium alloy to endodontics, there have been many changes in instrument design, but no significant improvements in the raw material properties, or enhancements in the manufacturing process. Recently, a new manufacturing process was developed by SybronEndo (Orange, CA), to create a NRT instrument the

*Address correspondence to this author at the Department of Dental Science, Sapienza University of Rome Dental School, Via Caserta 8, 00161 Rome, Italy; Tel: 003906 49976634; Fax: 00390649976627 1;

E-mail: ggambarini@tin.it
Twisted File (TF). It utilizes twisting of a ground blank in combination with heat treatment, to reportedly enhance super-elasticity and increase cyclic fatigue resistance.

To date, there have been few studies published to date on the mechanical properties of TF instruments [8-10]. The purpose of the present study was to investigate whether cyclic fatigue resistance is increased for nickel-titanium rotary (NTR) instruments manufactured using the new twisting process. This was evaluated by comparing $\mathrm{TF}$ the to NTR instruments produced by a traditional grinding process.

\section{MATERIALS AND METHODOLOGY}

In the present study four different NTR instruments from different manufacturers, of identical sizes (.06 taper and 0.25 tip diameter) were selected and evaluated:

1. TF (SybronEndo, Orange, CA, USA).

2. M2 (Sweden-Martina, Padova, Italy).

3. Profile (Maillefer, Baillagues, Switzerland).

4. Flexmaster (VDW, Munich, Germany).

Ten instruments from each manufacturer were tested for cyclic fatigue resistance, resulting in a total of 40 instruments. All instruments had been previously inspected using a measuring microscope for dimensional analysis, and for any signs of visible deformation. None was discarded. All tested instruments, with the exception of $\mathrm{TF}$, were produced using a traditional grinding process. Size $06-25$ was selected, being the master apical NTR file in many operative sequences.

The cyclic fatigue testing device used in the present study has been utilized in previous studies on cyclic fatigue resis- 
tance performed by the authors [8, 11, 12]. The device consists of a main frame to which a mobile plastic support is connected for the electric handpiece, and a stainless steel block containing the artificial canals (Fig. 1). The electric handpiece was mounted upon a mobile device to allow precise and reproducible placement of each instrument inside the artificial canal. This ensured three-dimensional alignment, and positioning of the instruments to the same depth. The artificial canal was manufactured by reproducing an instrument's size and taper. It provided the instrument with a suitable simulated root canal with a 60 degree angle of curvature and $5 \mathrm{~mm}$ radius of curvature. The centre of the curvature was $6 \mathrm{~mm}$ from the tip of the instrument and the curved segment of the canal was approximately $6 \mathrm{~mm}$ in length. The instruments were rotated at a constant speed of $300 \mathrm{rpm}$ using a 16:1 reduction handpiece (W \& $\mathrm{H}$ Dentalwerk, Burmoos, Austria), powered by a torque controlled electric stepper motor (X-Smart; Dentsply Maillefer, Ballaigues, Switzerland). Torque was set at $2 \mathrm{~N} / \mathrm{cm}$.

All instruments were rotated until fracture occurred. The time to fracture was recorded visually with a $1 / 100$-second chronometer. The time to fracture was multiplied by the number of rotations per minute (RPM), to obtain the number of cycles to failure (NCF) for each instrument. The length of the fractured tip was also recorded for each instrument.

Means and standard deviations (SD) of NCF and fragment length were calculated for each system. Data were subjected to one-way analysis of variance (ANOVA) to determine significant differences between groups. When the overall $F$-test indicated a significant difference, the multiplecomparison Holm $t$-test procedure was performed to identify the mean that differed from the others. Significance was set at the $95 \%$ confidence level.

\section{RESULTS}

Mean values \pm standard deviation expressed as NCF are displayed in Table 1. A higher number of cycles to failure is due to a higher resistance to cyclic fatigue of the tested instruments A statistically significant difference $(P<0.05)$ was noted between TF instruments and the other tested NTR instruments produced with a traditional grinding process. TF instruments, size 25, and.06 taper, showed the greatest mean number of cycles to failure. No statistically significant difference $(P>0.05)$ was noted between ProFile, FlexMaster and M2 NTR instruments

Table 1. Fatigue Resistance (Mean Number of Cycles to Failure and SD) of Size 25, Taper.06 Instruments

\begin{tabular}{|c|c|c|c|c|}
\hline \multirow{2}{*}{ Instruments } & \multicolumn{2}{|c|}{ NCF } & \multicolumn{2}{c|}{ Fragment Length } \\
\cline { 2 - 5 } & Mean & SD & Mean & SD \\
\hline \hline TF 25.06 & $770,01 *$ & 123,77 & $5,29 \xi$ & 0,40 \\
\hline M2 25.06 & 492,51 & 91,54 & $5,33 \xi$ & 0,25 \\
\hline FlexMast 25.06 & 555,83 & 83,47 & $4,58 \xi$ & 0,56 \\
\hline Profile 25.06 & 564,00 & 22,71 & $5,00 \xi$ & 0,58 \\
\hline
\end{tabular}

* Significant differences in NCF between TF and the other NTR instruments $(\mathrm{P}<$ $0.05)$.

No significant differences in NCF between M2, FlexMast and Profile $(P>0.05)$. $\xi$ No significant differences in fragment length between all NTR instruments $(P>$ $0.05)$.

Mean length of the fractured segment was also recorded to evaluate the correct positioning of the tested instrument

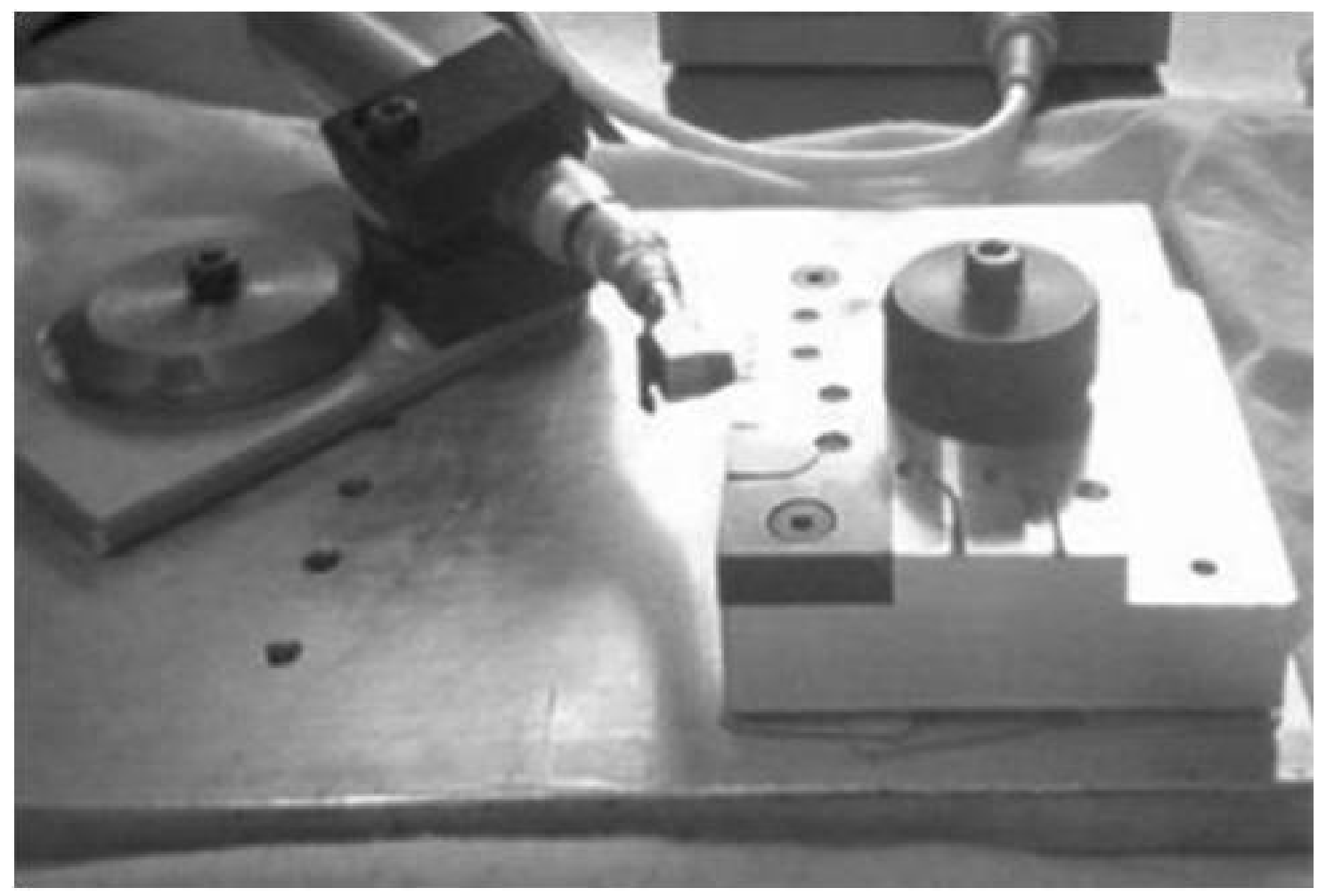

Fig. (1). The testing device. 
inside the canal curvature, and whether similar stresses were being induced. No statistically significant difference $(P>$ $0.05)$ in the mean length of the fractured fragments was evident for all of the instruments

\section{DISCUSSION}

There have been no scientific methods developed to date, which evaluate the resistance to fatigue of NTR instruments. Differences in testing devices used for evaluation can lead to different results, but so far there has been no device or method for fatigue testing incorporated into international standards for endodontic instruments. Furthermore, there is no testing protocols approved by the International Standard Organization (ISO) to establish minimum standards for an instrument's cyclic fatigue resistance. Nevertheless, with cyclic fatigue failure appearing to be the main mechanism leading to intracanal failure, fatigue testing of NiTi rotary instruments is probably the most useful tool to understand an instruments' fatigue properties. The cyclic fatigue testing device used in the present study has been utilized in previous studies of cyclic fatigue resistance $[8,11,12]$. The purpose of the present study was to investigate whether cyclic fatigue resistance is increased for nickel-titanium rotary (NTR) instruments manufactured using the new twisting process. This was evaluated by comparing TF NTR instrument produced by the process of heating and twisting to NTR instruments produced by traditional grinding methods. Results showed that size 06-25 TF instruments were significantly more resistant to cyclic fatigue than the other tested size 06-25 NTR instruments.

In a recent study [13] it has been determined that the fatigue life of NTR instruments is not significantly affected by differences in cross-sectional shape. Therefore, the improvement in cyclic fatigue resistance for size 06-25 TF instruments appears to be related to the manufacturing process. Moreover, it has been shown [14] that the design of the cutting flutes does not influence the fatigue resistance of instruments that are the same size.

The TF technology was developed to increase NTR instrument resistance to cyclic fatigue, and provide superior mechanical properties. The present in vitro evaluation of resistance to cyclic fatigue provided promising results. The innovative manufacturing process developed by SybronEndo, involving twisting a ground blank and heat treating it, produced a new instrument (TF) significantly more resistant to fatigue than NTR instruments produced with the traditional grinding process.

Results can be explained by the fact that the traditional grinding process has many drawbacks [15]. More precisely, cutting across the grain of the crystalline structure of the nickel-titanium wire limits the overall strength of the instruments, due to the formation of micro-cracks and defects along the surface of the instruments and within the internal structure [16-18]. These defects can cause stress concentration points that weaken the instruments and can lead to intracanal failure. Moreover cracks can propagate to failure at a stress level lower than the stress usually encountered during canal instrumentation, leading to sudden, unexpected file breakage $[19,20]$.

The preliminary findings of the present study must be confirmed by further investigations, which should evaluate other clinically relevant mechanical properties of the TF instruments. Additional studies are also necessary to improve correlations between in vitro and in vivo fatigue resistance of NTR instruments.

\section{CONCLUSION}

It can be concluded that the new twisting process produced size 06-25 NTR instruments (TF) significantly more resistant to fatigue than those produced with the traditional grinding process.

\section{ABBREVIATIONS}

NTR $=$ Nickel-Titanium Rotary
TF $=$ Twisted File
NCF $=$ Number of Cycles to Failure
SD $=$ Standard Deviation

\section{REFERENCES}

[1] Walia HM, Brantley WA, Gerstein H. An initial investigation of the bending and torsional properties of Nitinol root canal files. J Endod 1988; 14(7): 346-51.

[2] Kazemi RB, Stenman E, Spångberg LS. A comparison of stainless steel and nickel-titanium H-type instruments of identical design: torsional and bending tests. Oral Surg Oral Med Oral Pathol Oral Radiol Endod 2000; 90(4): 500-6.

[3] Xu X, Eng M, Zheng Y, Eng D. Comparative study of torsional and bending properties for six models of nickel-titanium root canal instruments with different cross-sections. J Endod 2006; 32(4): 3725 .

[4] Anderson ME, Price JW, Parashos P. Fracture resistance of electropolished rotary nickel-titanium endodontic instruments. J Endod 2007; 33(10): 1212-6.

[5] Fife D, Gambarini G, Britto Lr L. Cyclic fatigue testing of ProTaper NiTi rotary instruments after clinical use. Oral Surg Oral Med Oral Pathol Oral Radiol Endod 2004; 97(2): 251-6.

[6] Parashos P, Gordon I, Messer HH. Factors influencing defects of rotary nickel-titanium endodontic instruments after clinical use. $\mathrm{J}$ Endod 2004; 30(10): 722-5.

[7] Gambarini G. Cyclic fatigue of nickel-titanium rotary instruments after clinical use with low-and high-torque endodontic motors. J Endod 2001; 27(12): 772-4.

[8] Gambarini G, Grande NM, Plotino G, et al. Fatigue resistance of engine-driven rotary nickel-titanium instruments produced by new manufacturing methods. J Endod 2008; 34(8): 1003-5.

[9] Gambarini G, Gerosa R, De Luca M, Garala M, Testarelli L. Mechanical properties of a new and improved nickel-titanium alloy for endodontic use: an evaluation of file flexibility. Oral Surg Oral Med Oral Pathol Oral Radiol Endod 2008; 105(6): 798-800.

[10] Gambarini G, Pongione G, Rizzo F, Testarelli L, Cavalleri G, Gerosa R. Bending properties of nickel-titanium instruments: a comparative study. Minerva Stomatol 2008; 57(9): 393-8.

[11] Plotino G, Grande NM, Sorci E, Malagnino VA, Somma F. A comparison of cyclic fatigue between used and new Mtwo Ni-Ti rotary instruments. Int Endod J 2006; 39(9): 716-23.

[12] Grande NM, Plotino G, Pecci R, Bedini R, Malagnino VA, Somma F. Cyclic fatigue resistance and three-dimensional analysis of instruments from two nickel-titanium rotary systems. Int Endod J 2006; 39(10): 755-63.

[13] Cheung GS, Darvell BW. Low-cycle fatigue of NiTi rotary instruments of various cross-sectional shapes. Int Endod J 2007; 40(8): 626-32.

[14] Chaves Craveiro de Melo M, Guiomar de Azevedo Bahia M, Lopes Buono VT. Fatigue resistance of engine-driven rotary nickeltitanium endodontic instruments. J Endod 2002; 28(11): 765-9.

[15] Mc Spadden JT. Mastering Endodontic Instrumentation. Chattanooga TN: Cloudland Institute 2007. 
[16] Tripi TR, Bonaccorso A, Condorelli GG. Cyclic fatigue of different nickel-titanium endodontic rotary instruments. Oral Surg Oral Med Oral Pathol Oral Radiol Endod 2006; 102(4): e106-14.

[17] Yao JH, Schwartz SA, Beeson TJ. Cyclic fatigue of three types of rotary nickel-titanium files in a dynamic model. J Endod 2006; 32(1): 55-7.

[18] Gambarini G. Cyclic fatigue of profile rotary instruments after prolonged clinical use. Int Endod J 2001; 34(5): 386-9.
[19] Bahia MG, Melo MC, Buono VT. Influence of cyclic torsional loading on the fatigue resistance of $\mathrm{K} 3$ instruments. Int Endod J 2008; 41(10): 883-91.

[20] Johnson E, Lloyd A, Kuttler S, Namerow K. Comparison between a novel nickel-titanium alloy and 508 nitinol on the cyclic fatigue life of ProFile 25/.04 rotary instruments. J Endod 2008; 34(11): 1406-9.

(C) Testarelli et al.; Licensee Bentham Open.

This is an open access article licensed under the terms of the Creative Commons Attribution Non-Commercial License (http: //creativecommons.org/licenses/ by-nc/3.0/) which permits unrestricted, non-commercial use, distribution and reproduction in any medium, provided the work is properly cited. 